\title{
Quantitative analysis of Hedgehog gradient formation using an inducible expression system Vivian F Su ${ }^{1,2}$, Kelly A Jones ${ }^{1}$, Michael Brodsky*1 and Inge The ${ }^{* 1,3}$
}

Address: ${ }^{1}$ Program in Gene Function and Expression, Department of Molecular Medicine, University of Massachusetts Medical School, Worcester, MA, USA, ${ }^{2}$ Cancer Research Center, University of Hawaii at Manoa, Honolulu, HI, USA and ${ }^{3}$ Department of Developmental Biology, Utrecht University, Utrecht, the Netherlands

Email: Vivian F Su - vsu@crch.hawaii.edu; Kelly A Jones - kdonovan26@hotmail.com; Michael Brodsky* - michael.brodsky@umassmed.edu; Inge The* - s.i.the@uu.nl

* Corresponding authors

Published: 7 May 2007

BMC Developmental Biology 2007, 7:43 doi:10.1 I86/I47I-2I3X-7-43

This article is available from: http://www.biomedcentral.com/I47I-2/3X/7/43

This is an Open Access article distributed under the terms of the Creative Commons Attribution License (http://creativecommons.org/licenses/by/2.0), which permits unrestricted use, distribution, and reproduction in any medium, provided the original work is properly cited.

\begin{abstract}
Background: The Hedgehog $(\mathrm{Hh})$ family of secreted growth factors are morphogens that act in development to direct growth and patterning. Mutations in human $\mathrm{Hh}$ and other Hh pathway components have been linked to human diseases. Analysis of $\mathrm{Hh}$ distribution during development indicates that cholesterol modification and receptor mediated endocytosis affect the range of $\mathrm{Hh}$ signaling and the cellular localization of $\mathrm{Hh}$.
\end{abstract}

Results: We have used an inducible, cell type-specific expression system to characterize the threedimensional distribution of newly synthesized, GFP-tagged $\mathrm{Hh}$ in the developing Drosophila wing. Following induction of Hh-GFP expression in posterior producing cells, punctate structures containing Hh-GFP were observed in the anterior target cells. The distance of these particles from the expressing cells was quantified to determine the shape of the Hh gradient at different time points following induction. The majority of cholesterol-modified Hh-GFP was found associated with cells near the anterior/posterior (A/P) boundary, which express high levels of $\mathrm{Hh}$ target genes. Without cholesterol, the Hh gradient was flatter, with a lower percentage of particles near the source and a greater maximum distance. Inhibition of Dynamin-dependent endocytosis blocked formation of intracellular $\mathrm{Hh}$ particles, but did not prevent movement of newly synthesized $\mathrm{Hh}$ to the apical or basolateral surfaces of target cells. In the absence of both cholesterol and endocytosis, Hh particles accumulated in the extracellular space. Staining for the Hh receptor Ptc revealed four categories of Hh particles: cytoplasmic with and without Ptc, and cell surface with and without Ptc. Interestingly, mainly cholesterol-modified $\mathrm{Hh}$ is detected in the cytoplasmic particles lacking Ptc.

Conclusion: We have developed a system to quantitatively analyze $\mathrm{Hh}$ distribution during gradient formation. We directly demonstrate that inhibition of Dynamin-dependent endocytosis is not required for movement of $\mathrm{Hh}$ across target cells, indicating that transcytosis is not required for $\mathrm{Hh}$ gradient formation. The localization of $\mathrm{Hh}$ in these cells suggests that $\mathrm{Hh}$ normally moves across both apical and basolateral regions of the target cells. We also conclude that cholesterol modification is required for formation of a specific subset of $\mathrm{Hh}$ particles that are both cytoplasmic and not associated with the receptor Ptc. 


\section{Background}

Members of the Hh family have an evolutionarily conserved role in regulating growth and differentiation during development of many organisms [1]. Hh directly induces different cell fates in a concentration dependent manner, and thus is classified as a morphogen. This concentration gradient is tightly regulated and any disruption can cause abnormal cell specification [2,3]. Mutations in Hh pathway components have also been shown to lead to human disorders and disease [4].

In Drosophila, the Hh morphogen is produced and secreted from posterior compartment cells in embryos and imaginal wing discs. Hh travels to anterior target cells and forms a concentration gradient from its source $[1,5]$. Models that have been proposed for morphogen distribution and gradient formation include extracellular movement by diffusion and intracellular movement by transcytosis $[4,5]$. The diffusion model can be further divided into the free diffusion and restricted planar diffusion models. In the free diffusion model, the morphogen is secreted from the producing cells into the extracellular space and diffuses in three-dimensions out to the target cells. In the restricted planar diffusion model, the morphogen moves directly from cell to cell, always remaining in the two-dimensional epithelial cell layer close to the cell surface. In contrast, the transcytosis model proposes a unique mechanism where successive rounds of endocytosis and exocytosis move Hh through cells $[4,5]$.

Hh proteins in all organisms are dually lipid modified as part of their intracellular processing to produce $\mathrm{HhNp}$ (p for processed) and these modifications are likely to affect movement of the morphogen. In Drosophila, Hh is synthesized as a $45 \mathrm{kDa}$ full length precursor protein that undergoes an autoproteolytic cleavage [6,7]. Cholesterol is covalently attached to the $\mathrm{C}$-terminus of the $\mathrm{N}$-terminal signaling molecule as part of this process [8]. A palmitoyl group is attached at the $\mathrm{N}$-terminus by a membrane bound O-acyltransferase to produce a dually lipidated 19 $\mathrm{kDa}$ HhNp molecule [9-13]. Because the protein is lipid modified, Hh movement must include a mechanism that prevents this modification from restricting $\mathrm{Hh}$ to the membranes of the producing cells. One mechanism to mobilize lipid modified Hh may be to form micelle-like structures; in gel filtration assays, Hh and vertebrate Sonic Hedgehog (Shh) multimers, which require both lipid modifications, can be detected $[14,15]$. This high molecular weight $\mathrm{Hh}$ fraction will associate with cell membranes in tissue culture cells while the monomeric forms do not $[16,17]$. The hydrophobic moieties could be hidden inside multimeric micelle-like structures to make the HhNp complexes more soluble in order to diffuse $[15,18]$. Therefore, the cholesterol modification could be required for multimerization that enables long range movement and proper gradient formation. The requirement for cholesterol modification in signaling is not clear due to conflicting reports from both Drosophila and mammalian studies. In some studies, using Drosophila wing discs and mouse limb buds, the unmodified $\mathrm{Hh}(\mathrm{HhN})$ has long range activity $[8,14,19-21]$. However, other Drosophila and mammalian studies suggest that cholesterol is required for long range activity [16,22-24].

In Drosophila and vertebrates, the extracellular matrix components Heparan Sulfate Proteoglycans (HSPGs) are involved in Hh movement. Loss of HSPGs block Hh movement and signaling in adjacent wild-type cells is impaired [25-31]. Interestingly, HSPG regulation of $\mathrm{Hh}$ movement depends on cholesterol, as unmodified $\mathrm{HhN}$ is unaffected by the loss of HSPGs [14]. The cholesterol may mediate Hh and HSPG association, as cholesterol-modified $\mathrm{Hh}$ and Shh are able to bind heparin [6,32]. One interpretation of these results is that HSPGs are required to mediate planar diffusion of cholesterol-modified $\mathrm{Hh}$ across or through target cells, but that unmodified $\mathrm{Hh}$ is able to move via free diffusion. In support of this model, unmodified Hh expressed in the peripodial cell layer of the developing Drosophila wing can move through the extracellular space of the wing lumen while cholesterolmodified $\mathrm{Hh}$ is restricted to the layer of cells in which it is expressed [14,22].

Receptor-mediated endocytosis has also been proposed to regulate the spreading of $\mathrm{Hh}$. In addition to transducing the Hh signal, Patched (Ptc), the receptor for $\mathrm{Hh}$, has been shown to sequester and limit the range of distribution by binding and internalizing $\mathrm{Hh}[33,34]$. Hh is thought to be primarily endocytosed together with Ptc and then targeted for degradation [14], although Ptc-independent cytoplasmic Hh particles have been detected as well [34,35]. A role for endocytosis in Hh gradient formation has been proposed, either as part of transcytosis or by removing Hh to limit the distribution range. Blocking endocytosis in embryos and wing discs with a dynamin mutant (shibire in Drosophila or shi) does not appear to affect Hh target gene expression or spreading $[14,16,28,34]$. These observations suggest that Dynamin-mediated endocytosis may be required for $\mathrm{Hh}$ degradation but not $\mathrm{Hh}$ distribution. However, in these experiments, shi is inactivated in tissues with a preexisting gradient of $\mathrm{Hh}$. It is unclear whether the Hh distribution observed reflects this preexisting pool or newly synthesized Hh produced following shi inactivation.

While previous studies provided much information about Hh distribution, these studies also have some limitations that may contribute to conflicting conclusions. One limitation of previous studies was that overexpressed $\mathrm{Hh}$ and/ or preexisting pools of $\mathrm{Hh}$, instead of newly produced 
protein, were examined [36]. Therefore, the observed distribution of Hh may reflect redistribution of preexisting protein instead of the ability of Hh to move despite the endocytosis block. This issue can be addressed by using an inducible system where the movement of newly synthesized protein is studied. Another limitation is that Hh distribution was examined after the Hh gradient had reached a steady state; analysis during gradient formation may provide more information about Hh movement. This too can be addressed with an inducible system. Finally, the ability to determine the magnitude and statistical significance of changes in Hh distribution may be limited by the potential difficulty in measuring the three-dimensional distance traveled from the producing cells.

We have attempted to resolve these issues by quantitatively investigating the distribution of GFP-tagged forms of Hh protein using an inducible expression system. To determine whether the cholesterol modification and/or endocytosis have any effect on the process of gradient formation, we expressed functional Hh-GFP with and without the cholesterol-modification in a wild-type or shits1 mutant background. Specifically, the Gal80-Gal4 system was used to temporally express (pulse) Hh-GFP transgenes in their normal expression domain (the posterior compartment of the developing wing); this method allows the rate of Hh gradient formation over time to be observed. We demonstrate that both HhNp-GFP and HhN-GFP are present in punctate structures (particles). We have also developed a system for quantitative measurement of Hh distribution and found that HhN-GFP migrates further than HhNp-GFP while less is retained near the expressing cells. We demonstrate that when endocytosis is blocked, newly synthesized HhNp-GFP is still detected in particles and can still move anteriorly, arguing against an essential role of transcytosis. Lumenal HhN-GFP particles were observed, indicating cholesterol is responsible for retaining $\mathrm{Hh}$ on cell surfaces. We propose that HhNp and HhN spread through both apical and basolateral regions by planar diffusion and that the cholesterol modification serves to retain $\mathrm{Hh}$ on the cell surface and promotes the formation of a steep gradient.

\section{Results \\ Generation of Hh-GFP fusion constructs and functional characterizations}

To study movement and distribution of newly synthesized $\mathrm{Hh}$, we generated Hh-GFP fusion proteins that are suitable for both live and fixed tissue experiments. The HhFGFP fusion construct (Figure 1A) contains GFP coding sequences placed between $\mathrm{Hh}$ amino acids $254(\mathrm{H})$ and $255(\mathrm{~V})$. The same location was used previously to generate functionally tagged $\mathrm{HhNp}[14,19,34]$, which is expected to be processed into a HhNp-GFP and an untagged Hh-C. To express unprocessed HhN-GFP, we generated an expression construct that encodes the N-terminus of Hh fused to GFP, similar to a HhN-GFP fusion described previously [37].

Several experiments were performed to determine whether HhNp-GFP is functional. First, HhNp-GFP was constitutively expressed with the Gal4-UAS system [38] using Hh-Gal4, which would express the transgene using the endogenous $h h$ promoter. DsRed protein was coexpressed with the Hh fusion protein to identify expressing cells and mark the anterior/posterior (A/P) boundary in this and subsequent experiments. HhNp-GFP was secreted from the dsRed-expressing cells similar to untagged wild-type HhNp (Figure 1B-D). Second, HhNpGFP and HhN-GFP were expressed in salivary glands using the ubiquitous 71B-Gal4 driver and the glands were extracted to identify the Hh fusion proteins on a Western blot (Figure 1E). For HhN-GFP, an approximately $46 \mathrm{kDa}$ protein band was detected corresponding to the predicted size of the N-terminus fused to GFP (lane 3). For HhNp, an approximately $70 \mathrm{kDa}$ band representing the uncleaved full length HhF-GFP protein (U) and a $46 \mathrm{kDa}$ band representing the cleaved HhNp-GFP signaling molecule $(\mathrm{P}$, lane 4$)$ were observed. These results indicate that both Hh-GFP fusion proteins are expressed and properly processed. Third, we tested the ability of HhNp-GFP to rescue the embryonic lethality of homozygous $h h^{G S 1}$ mutants. Expressing HhNp-GFP in the posterior compartment using the En-Gal4 driver, we were able to fully rescue $h h^{G S 1}$ mutants to adulthood. These animals appeared to develop normally, as demonstrated by a normal though slightly smaller wing (Figure 1G). Finally, wings of flies ectopically expressing functional untagged $\mathrm{HhNp}$ [7] and wings expressing HhNp-GFP had similar phenotypes of merged wing veins L2 and L3 (Figure 1H and 1I). These results demonstrate that the Hh-GFP fusion proteins are properly synthesized, and that HhNp-GFP has the same properties as previously described for wild-type $\mathrm{HhNp}$ [39].

\section{Analysis of HhNp-GFP localization in living tissue}

The Drosophila larval wing imaginal disc consists of two layers of epithelial cells, separated by the peripodial lumen. On the apical side, a squamous epithelial layer forms the peripodial membrane, while on the basal side, the disc epithelium is found that will give rise to the wing and notum. Most previous studies exploring HhNp localization in the disc epithelium have been performed with fixed discs. In these studies and our own experiments with fixed discs, most HhNp in the anterior compartment is found in punctate structures, as detected by immunostaining. To rule out the possibility of fixation-induced alterations in Hh localization, we took advantage of the GFP tag to examine the localization of HhNp-GFP in live tissues. Hh-Gal4 was used to express HhNp-GFP in the endog- 
A

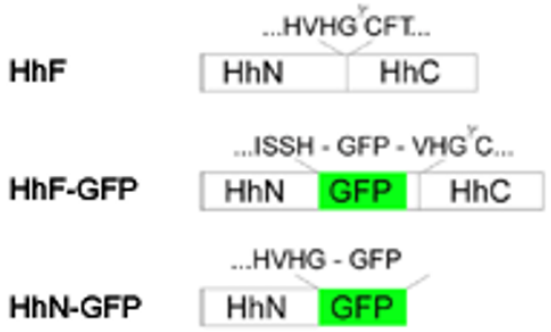

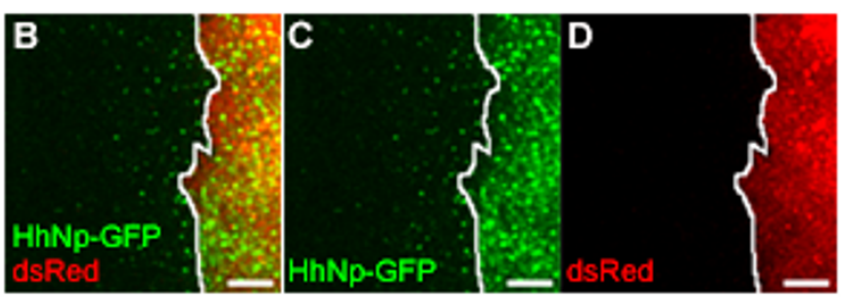
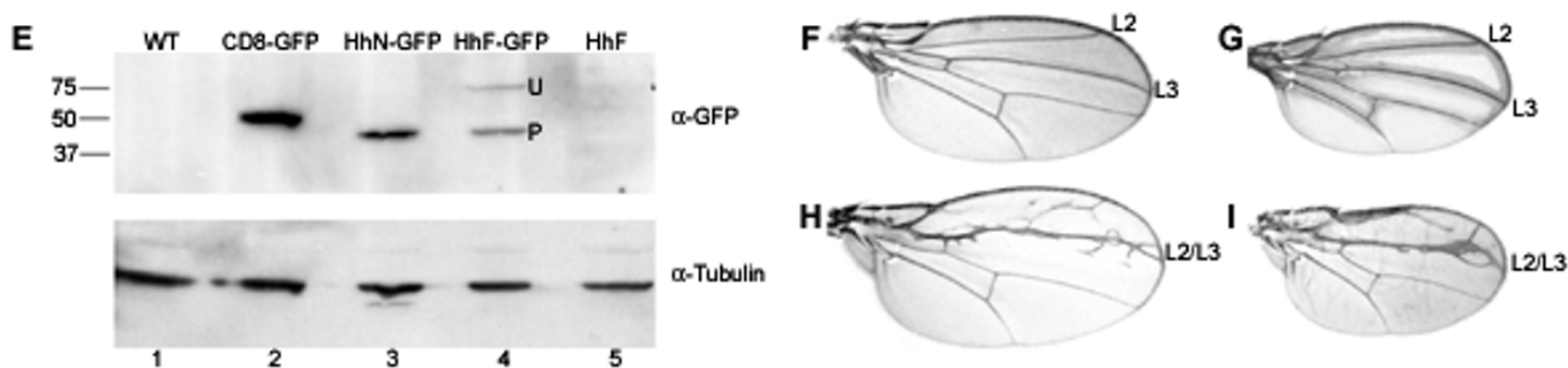

Figure I

HhNp-GFP is functional. (A) Scheme of HhF-GFP (middle) and HhN-GFP (bottom) fusion constructs and predicted processing as compared to wild-type HhF (top). HhF-GFP is predicted to be processed into HhNp-GFP. (B-D) HhNp-GFP (green, C) is expressed in posterior cells labeled by fluorescent protein dsRed (red, D) and secreted (B), similar to wild-type $\mathrm{HhNp}(\mathrm{A} / \mathrm{P}$ boundary marked by a solid white line). Scale bar: $5 \mu \mathrm{m}(\mathrm{E})$ Western blot of salivary gland protein extracts labeled with anti-GFP (upper panel) or tubulin (lower panel). Upper panel: as negative controls we used extracts of wild-type w 1118 larvae (lane I) and larvae expressing an untagged $\mathrm{HhF}$ (lane 5), CD8-GFP was used as a positive control (lane2). A single $46 \mathrm{kDa}$ band is seen in the lane with HhN-GFP expressing larvae (lane 3) and in the lane loaded with extract of HhF-GFP expressing larvae, two bands of $70 \mathrm{kDa}$ and $46 \mathrm{kDa}$ are seen (lane 4; U: unprocessed full-length HhF-GFP, P: processed HhNp-GFP). Lower panel: the same blot was reprobed with anti-tubulin for loading control. (F-I) HhF expression in adult wings. (F) Wildtype wing. (G) Wing from HhF-GFP rescue of $h h^{G S I}$ mutant has a similar phenotype to wild-type. $(H)$ As a positive control, untagged $\mathrm{HhF}$ is expressed with 7IB-Gal4 resulting in merged veins L2 and L3. (I) Ectopic expression of HhF-GFP has a similar phenotype to untagged HhF.

enous Hh expression domain in the posterior compartment of the wing imaginal disc [40]. In live discs, HhNpGFP co-localized with the membrane marker FM4-64 in the posterior compartment and was also found in particles in both the posterior and anterior compartments (Figure $2 \mathrm{~A}$ ). These particles were found in both apical and basolateral regions (Figure 2B). HhNp has previously been observed in endosomes [14,16,34,35]. We used endocytosed dextran to determine whether the particles containing HhNp-GFP in live discs included endosomes. In both the anterior and posterior compartments, many, but not all, of the HhNp-GFP particles co-localized with dextran (Figure 2C and 2D), confirming that at least some HhNp particles correspond to endosomes. The non-colocalizing HhNp-GFP vesicles could represent endosomes formed before or after dextran incubation, non-endocytic vesicles, or extracellular particles.

We then investigated the extracellular distribution of HhNp-GFP, using an "in vivo" extracellular labeling method as described by Strigini and Cohen [41]. In this procedure, live discs are incubated with anti-GFP antibody and then washed prior to fixation and detergent treatment. In control experiments using this method, we detected a protein with an extracellular GFP tag (GFPDally-like) while an intracellular YFP tag (Ptc-YFP) was not detected, demonstrating that the staining procedure reliably distinguished between extracellular and intracellular localization (Additional File 1). When similar experiments were performed for HhNp-GFP, strong extracellular staining was observed in the apical and basal regions in the posterior compartment of the disc where $\mathrm{Hh}$ is produced (Figure 2E). In the anterior compartment, extracellular HhNp-GFP was detected in apical particles (Figure 2E, arrows), basolateral particles (Figure 2E, arrowheads) and on the basolateral membrane (Figure 2E, bracket). Extracellular HhNp-GFP was also detected in the overlying peripodial membrane. While some of these cells align with the anterior compartment of the disc proper cells, this region corresponds to the posterior com- 

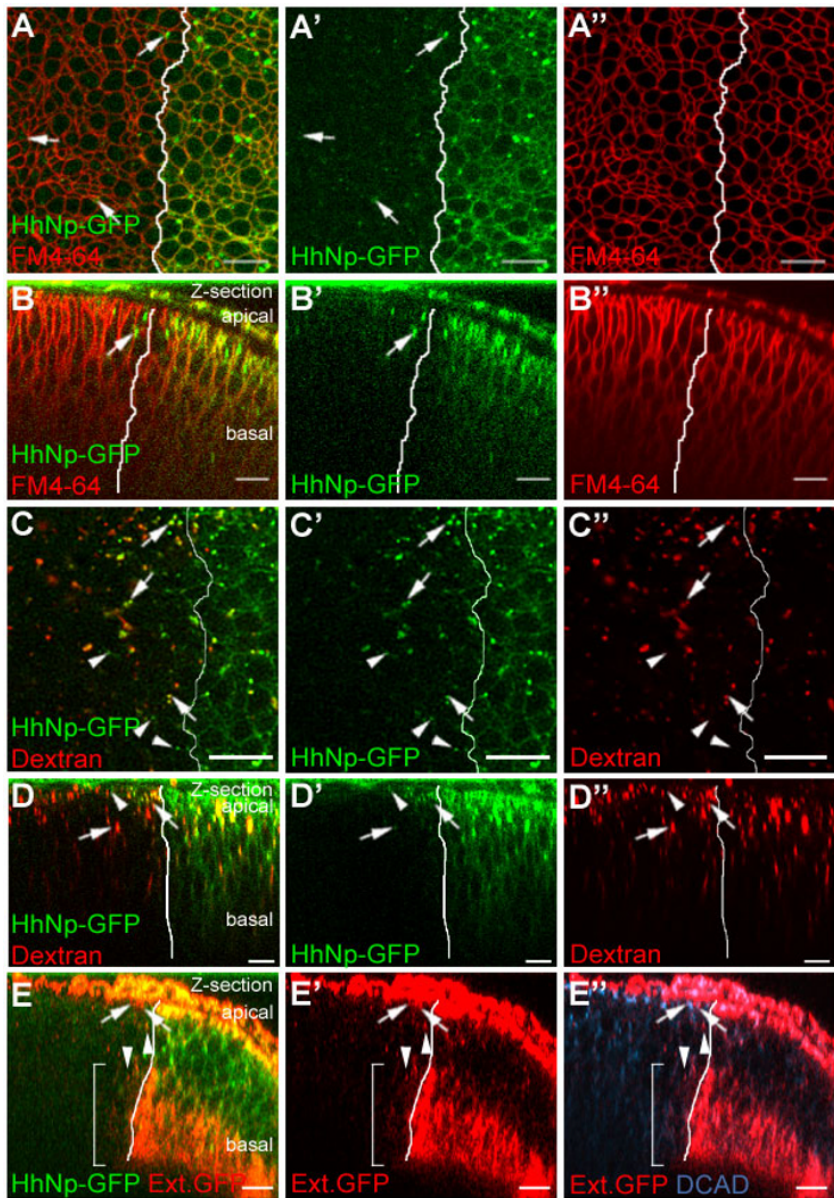

Figure 2

HhNp-GFP localizes at the membrane, in endocytic compartments, and extracellularly. (A-E) Localization of HhNp-GFP (green), expressed with Hh-Gal4, with FM4-64 (red, A-B), dextran (red, C-D), extracellular labeling with anti-GFP, and DCAD (red and blue, E). (B, D, E) Z-sections. HhNp-GFP ( $\left.A^{\prime}\right)$ co-localizes with FM4-64 (A") at the membrane in the posterior (A/P boundary marked by the solid white line) as seen in the merge (A). Anterior HhNp-GFP appears in particles (arrows). Most of the particles localize apically (B). Many of the anterior HhNp-GFP particles colocalize (arrows in C-D) with dextran but some can be found without dextran (arrowheads in C-D). Incubation of antiGFP in cold medium detects extracellular HhNp-GFP in the anterior apically in particles (arrows in E), and basolaterally both in particles (arrowheads in E) and with a membrane association (bracket in E). Scale bar: $8 \mu \mathrm{m}$

partment of the peripodial membrane where HhNp-GFP is being expressed [42]. These localization results are consistent with previously published data for both untagged and tagged HhNp $[14,22,34]$. In conclusion, the largely punctate localization of tagged and native Hh observed in the anterior compartment of the developing wing after fixation accurately reflects the localization of $\mathrm{Hh}$ in living tissues.

\section{Induction and quantitative analysis of a Hh gradient}

In the experiments described above and by other groups, Hh distribution in the developing wing is characterized following expression over a period of several days. To examine the movement and distribution of newly synthesized $\mathrm{Hh}$, we used the Gal80-Gal4 temperature-sensitive inducible system [43]. At a lower temperature, Gal80 inhibits Gal4 activity (Figure 3A). Following a shift to higher temperature, Gal80 is inactivated and Hh-Gal4 activates expression of Hh-GFP in posterior wing cells. With this system, we were able to analyze Hh distribution at different time points following induction (Figure 4). At 8 hours following induction, Hh-GFP exists as particles in the anterior compartment, primarily near the A/P boundary. At 24 and 72 hours following induction, increased numbers of particles are observed, both near the $\mathrm{A} / \mathrm{P}$ boundary and further from the expressing cells (Figures 4, 5, and Additional File 2).

To examine how different factors alter the distribution of newly synthesized $\mathrm{Hh}$, we developed an assay to quantify the distance of individual particles of Hh-GFP from the A/ $P$ boundary. First, a series of optical sections were transformed into a three-dimensional reconstruction of $\mathrm{Hh}$ GFP and dsRed localization in a region of the wing disc near the A/P boundary (Figure 5A). Next, the dsRed expressing cells were converted into a single isosurface and the distance of Hh-GFP particles from this surface was determined (see Methods and Figure 3A-C, individual data sets are shown in Additional File 3). Because the number of particles induced was variable, the percentages of Hh-GFP particles at different distances from the $\mathrm{A} / \mathrm{P}$ boundary were used to normalize distributions profiles within and between different experimental conditions (Figure 5, Additional File 4).

To establish how long the Hh gradient takes to form (Figure 4 and 5), we analyzed the change in HhNp-GFP distribution at 8, 24, and 72 hours following induction (Figure $5 \mathrm{D}-\mathrm{F}$, Table 1 ). At each time point, the majority of $\mathrm{HhNp}$ GFP was detected within $8 \mu \mathrm{m}$ of the A/P boundary. This distance represents the average width of the region expressing high levels of the Hh target gene ptc (unpublished results). The percentage of HhNp-GFP particles in this region significantly decreased from 8 to 24 hours, but not from 24 to 72 hours (Table 2). Similarly, both the median and the $90^{\text {th }}$ percentile values for the HhNp-GFP distributions significantly increased from 8 to 24 hours, but not from 24 to 72 hours. From this analysis, we conclude that the HhNp-GFP gradient is still forming at 8 hours and is approaching its final shape by 24 hours.

\section{Cholesterol modification is required for proper $\mathrm{Hh}$ distribution}

Having identified time points when newly synthesized $\mathrm{HhNp}$ is forming a gradient ( 8 and 24 hours) or has 
A

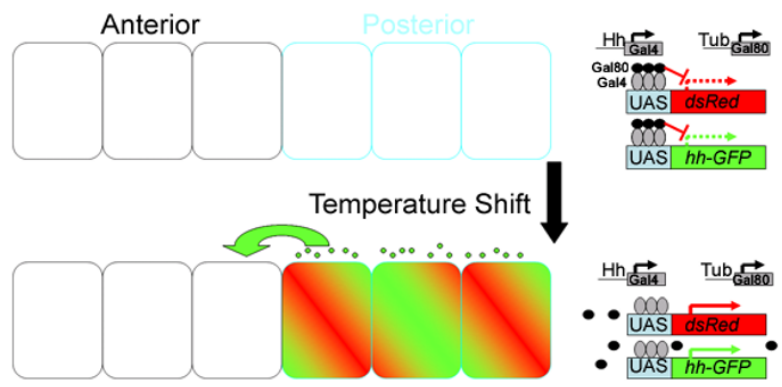

B

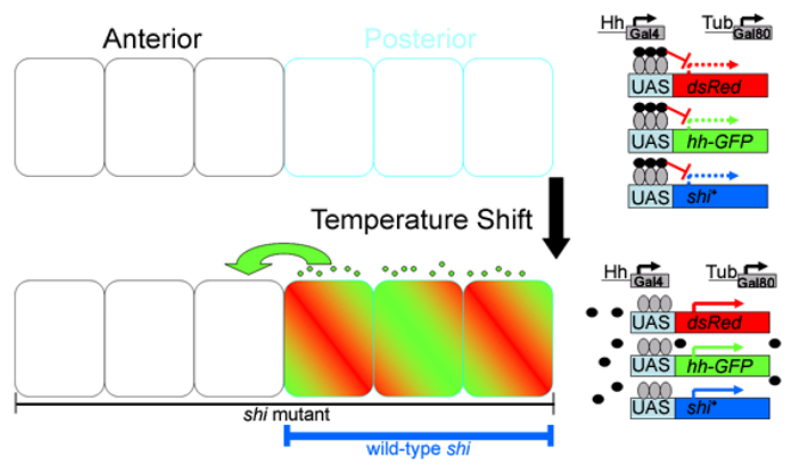

Figure 3

Schematic diagram of Gal4-Gal80 inducible expression system. (A) Inducing Hh-GFP expression. Vials are kept at $18^{\circ} \mathrm{C}$, the Gal80 permissive temperature where tubulin-Gal80 blocks Gal4-mediated transcription. Upon a shift to $32^{\circ} \mathrm{C}$, Gal80 repression is relieved and Gal4 transcription proceeds. Hh-GFP is expressed in the posterior cells with $\mathrm{Hh}-\mathrm{Gal} 4$, and UAS-dsRed marks the expressing cells. (B) Inducing $\mathrm{Hh}$-GFP expression in shits $\mathrm{mutant}$ background. Vials are kept at $18^{\circ} \mathrm{C}$, the Gal80 permissive temperature and shits I mutant restrictive temperature. Upon a shift to $32^{\circ} \mathrm{C}, \mathrm{Gal} 4$ transcription proceeds while endocytosis is blocked. Wild-type Shi is expressed in the posterior to restore endocytosis in the expressing cells. The resulting $\mathrm{Hh}$ GFP movement into the anterior would be due solely to shi independent mechanisms. $\mathrm{Hh}-\mathrm{Gal} 4$ is used again to drive transgene expression.

reached a steady state (72 hours), we examined the distribution of HhN-GFP, which lacks the cholesterol modification, at these same time points. Similar to HhNp-GFP, the majority of HhN-GFP was detected within the first 8 $\mu \mathrm{m}$ from the $\mathrm{A} / \mathrm{P}$ boundary (Table 1 ) and the median value, $90^{\text {th }}$ percentile, and percent of particles at $8 \mu \mathrm{m}$ changed from 8 to 24 hours, but not from 24 to 72 hours (Table 2). However, the shape of the gradient is different for $\mathrm{HhN}$ than HhNp. Comparisons of composite distribution profiles reveal that the distribution of HhN-GFP is shifted further from the $\mathrm{A} / \mathrm{P}$ boundary at all time points (Figures $5 \mathrm{E}-\mathrm{F}$ ). The median and $90^{\text {th }}$ percentile values are statistically significantly greater for HhN-GFP than for HhNp-GFP (Tables 1 and 2) indicating that $\mathrm{HhN}$ is able

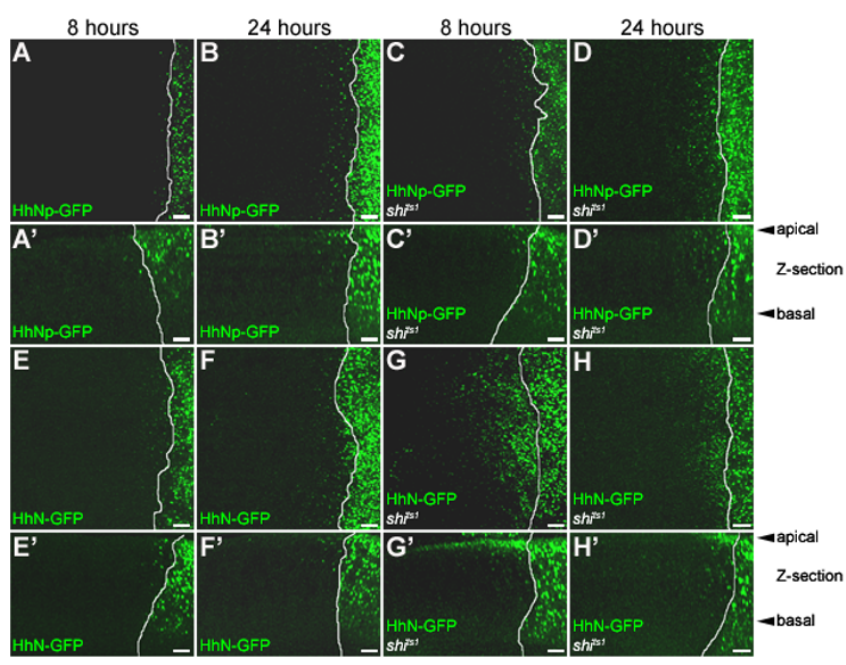

Figure 4

Cholesterol restricts $\mathrm{HhNp}$-GFP distribution but endocytosis is not required for distribution. $(A-H)$ Induced expression of HhNp-GFP in wild-type (A-B) and shits I background (C-D) and HhN-GFP in wild-type (E-F) and shits I background (G-H). (A-H) $25 \mu \mathrm{m}$ projections; $\left(\mathrm{A}^{\prime}-\mathrm{H}^{\prime}\right) 20$ $\mu \mathrm{m}$ Z-section projections. At $8 \mathrm{hr}, \mathrm{HhNp}$-GFP particles are found near the A/P boundary, marked by the solid white line $\left(A, A^{\prime}\right)$. After $24 \mathrm{hr}$, more particles can be found further away (B, B'). HhN-GFP particles are detected further from the A/P boundary than $\mathrm{HhNp}$-GFP at both time points (E-F). When endocytosis is blocked, $\mathrm{HhNp}$-GFP particles are still detected in anterior cells (C-D). In wild-type and shits I backgrounds, HhNp-GFP particles appear closer to the apical side (A'-D') as well as $\mathrm{HhN}$-GFP in wild-type. When endocytosis is blocked, HhN-GFP moves into the anterior but there is reduced punctate staining and more membrane accumulation $(G-H)$, primarily on the apical side of cells $\left(G^{\prime}-H^{\prime}\right)$. Scale bar: $5 \mu \mathrm{m}$

to move further from the producing cells. In addition, the percentage of HhN-GFP within $8 \mu \mathrm{m}$ was significantly lower (Tables 1 and 2). This quantitative analysis extends previous studies indicating that $\mathrm{HhN}$ is able to move further into the anterior compartment than $\mathrm{HhNp}$ $[14,19,20]$. This difference is not simply a result of greater amounts of HhN being secreted from producing cells. Rather, the cholesterol modification of Hh contributes to the shape of the gradient. Specifically, cholesterol is required to create a steeper Hh gradient with a higher percentage near the $\mathrm{A} / \mathrm{P}$ boundary and a decreased maximum distance traveled.

\section{Movement of newly synthesized HhNp-GFP particles does not require Shi}

Several studies have shown that Hh is internalized with Ptc and localizes in endocytic compartments through a mechanism that requires the Drosophila Dynamin homolog Shi $[14,28,34]$; transient inhibition of Shidependent endocytosis blocks Hh internalization, but 


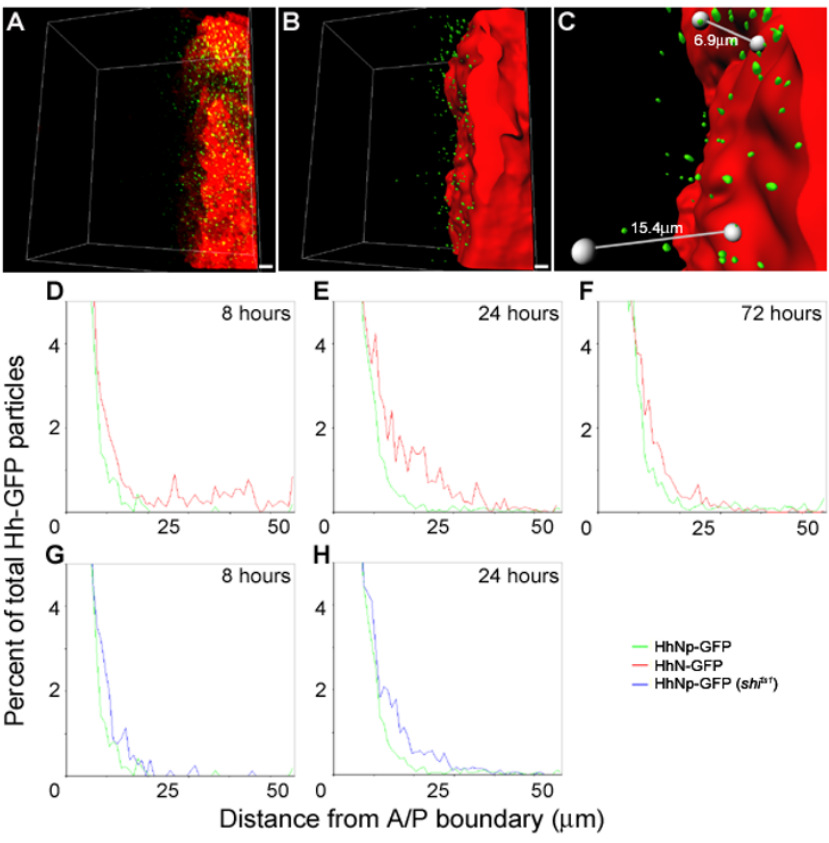

Figure 5

Quantitative analysis of Hh-GFP distribution: Cholesterol is required to restrict distribution but endocytosis is not required for distribution. (A-C) Schematic illustration of quantitative analysis. (A) Three dimensional reconstruction of a confocal z-stack with Hh-GFP (green) and dsRed (red) marking the expressing cells. (B) Generation of isosurfaces. DsRed isosurfacing was used to generate a distance map used to measure distances of Hh-GFP particles. Hh-GFP particles were isosurfaced to identify particles using an intensity threshold and size criteria. (C) Depiction of particle distance measurements. Particles were measured for the shortest distance to the expressing cells (lines depict manual measurements but all measurements were calculated in an automated fashion). Scale bar: $5 \mu \mathrm{m}$. (D-F) Mean of normalized HhNp-GFP (green) versus HhN-GFP (red) distribution profiles in a wild-type background. All samples were normalized to generate percentages of particles at the distances. Normalized data was then averaged to generate distribution profiles. Enlargement of the distribution near the $x$ axis shows more $\mathrm{HhN}$-GFP is detected further from the A/P boundary ( 0 on the $x$-axis) at 8 (D; HhNp-GFP $n=5, H h N$ GFP $\mathrm{n}=4)$ and $24 \mathrm{hr}(\mathrm{E} ; \mathrm{HhNp}-\mathrm{GFP} \mathrm{n}=16$, HhN-GFP $\mathrm{n}=$ $7)$. The same is seen at $72 \mathrm{hr}(\mathrm{F} ; \mathrm{HhNp}-\mathrm{GFP} \mathrm{n}=5$, HhN-GFP $n=6)$. (G-H) Mean of normalized HhNp-GFP distribution profiles in wild-type background (green) versus shits I mutant

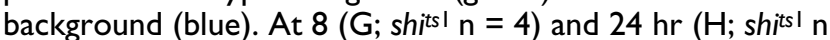
$=7$ ), $\mathrm{HhNp}$-GFP in the mutant background is less restricted and found further away from the A/P boundary than in the wild-type background. The same HhNp-GFP distribution profiles in the wild-type background from $D$ and $E$ are used for $\mathrm{G}$ and $\mathrm{H}$, respectively. does not affect Hh-dependent gene expression. These experiments may indicate that endocytosis is not required for movement of Hh into its target cells. However, because Hh is synthesized prior to Shi inhibition, it is not clear whether the Hh observed in target cells is newly synthesized or was present prior to shi inactivation.

To address this question, we used the inducible Hh-GFP system to simultaneously initiate a pulse of Hh-GFP expression in the posterior compartment and inhibit Shi function in the anterior compartment. Initially, we expressed HhNp-GFP throughout development (i.e. without Gal80) and then blocked endocytosis for 8 hours with the temperature sensitive mutation shits ${ }^{i}$; under these conditions, HhNp-GFP accumulated at the basal membranes of the anterior cells similar to previously published results $[14,28,34]$ and in more apical punctate structures (Additional File 5). Next, the effects of simultaneously inducing Hh-GFP expression while transiently blocking endocytosis were examined. In these experiments using the Gal80Gal4 system, the same temperature shift induces Hh-GFP and dsRed expression and inactivates $s h i{ }^{t s}$. In addition, wild-type Shi was also expressed in the posterior compartment, rescuing the endocytosis defect in the expressing cells (Figure 3B). Thus, Hh-GFP was induced in the posterior cells concurrently with a block in Shi-dependent endocytosis. Newly synthesized HhNp-GFP was observed in the anterior compartment even when shi function was simultaneously inactivated (Figure $4 \mathrm{C}^{\prime}$ and $4 \mathrm{D}^{\prime}$ ). The reduction of cytoplasmic particles of Hh-GFP confirms that endocytosis is inhibited in these experiments (Figure 6 , discussed below). These results directly demonstrate that Hh does not require Shi function to move into and across target cells in the anterior compartment. Based on these observations, we conclude that Shi-dependent transcytosis is not essential for movement of HhNp-GFP.

In contrast to the results with constitutively expressed HhNp-GFP which accumulated on the basolateral membranes of the anterior compartment in the absence of Shi [14], induced HhNp-GFP was predominantly found in particles and no basal membrane accumulation was observed (Figure 4). These particles could be detected at both 8 and 24 hours when Shi-dependent endocytosis is blocked (Figure 4C-D) and could be found in both apical and basolateral positions.

The distribution of HhNp-GFP particles in the absence of Shi function was quantified as described above. Overall, the distribution profiles are similar for HhNp-GFP in the wild-type and shits 1 mutant backgrounds (Figure 5 and Additional File 4) with the majority of HhNp-GFP found within $8 \mu \mathrm{m}$ of the $\mathrm{A} / \mathrm{P}$ boundary (Table 1 ). While the median values were not statistically significantly different in the shits1 mutant compared to a wild-type background, 
Table I: Analysis of Hh-GFP particle distribution in wing discs

\begin{tabular}{|c|c|c|c|c|}
\hline Time point & Sample & Median & $90^{\text {th }}$ percentile distance & $\%$ within $8 \mu \mathrm{m}$ \\
\hline \multirow[t]{3}{*}{$8 \mathrm{hr}$} & HhNp-GFP & $2.5 \mu \mathrm{m} \pm 0.6 \mu \mathrm{m}$ & $8 \mu \mathrm{m} \pm 1.4 \mu \mathrm{m}$ & $93 \pm 3.2$ \\
\hline & HhN-GFP & $4.4 \mu \mathrm{m} \pm 3.0 \mu \mathrm{m}$ & $27 \mu \mathrm{m} \pm 15.8 \mu \mathrm{m}$ & $75 \pm 18.4$ \\
\hline & HhNp-GFP(shitsl) & $2.7 \mu \mathrm{m} \pm 0.4 \mu \mathrm{m}$ & $10 \mu \mathrm{m} \pm 1.8 \mu \mathrm{m}$ & $85 \pm 6.4$ \\
\hline \multirow[t]{3}{*}{$24 \mathrm{hr}$} & HhNp-GFP & $3.5 \mu \mathrm{m} \pm 0.9 \mu \mathrm{m}$ & $\mathrm{II} \mu \mathrm{m} \pm 2.4 \mu \mathrm{m}$ & $82 \pm 7.7$ \\
\hline & HhN-GFP & $6.1 \mu \mathrm{m} \pm 1.9 \mu \mathrm{m}$ & $25 \mu \mathrm{m} \pm 7.0 \mu \mathrm{m}$ & $64 \pm 10.7$ \\
\hline & HhNp-GFP(shitsI) & $4.8 \mu \mathrm{m} \pm 1.2 \mu \mathrm{m}$ & $16 \mu \mathrm{m} \pm 4.8 \mu \mathrm{m}$ & $70 \pm 8.8$ \\
\hline \multirow[t]{2}{*}{$72 \mathrm{hr}$} & HhNp-GFP & $3.8 \mu \mathrm{m} \pm 0.6 \mu \mathrm{m}$ & $12 \mu \mathrm{m} \pm 2.1 \mu \mathrm{m}$ & $79 \pm 8.4$ \\
\hline & $\mathrm{HhN}-\mathrm{GFP}$ & $5.1 \mu \mathrm{m} \pm 1.2 \mu \mathrm{m}$ & $3 \mathrm{l} \mu \mathrm{m} \pm 3.7 \mu \mathrm{m}$ & $70 \pm 11.5$ \\
\hline
\end{tabular}

Between 4-16 discs were counted for each genotype and time point. Standard deviation was calculated for each measurement.

the percentage of particles within $8 \mu \mathrm{m}$ as well as the distance of the $90^{\text {th }}$ percentile was significantly different in the shits $^{1}$ mutant (Table 2). These results suggest that while HhNp-GFP movement through the anterior compartment is not drastically altered when Shi-mediated endocytosis is blocked, Shi function does contribute to the shape of the Hh gradient; following inhibition of Shi, the HhNpGFP gradient is less steep with a lower percentage of particles retained near the A/P boundary.

The distribution of newly synthesized HhN-GFP following shi inactivation was also examined. Under these conditions, HhN-GFP predominantly accumulated at the apical surface of the cells (Figure 4G-H), although some basolateral punctate structures were still detected. HhNGFP could also be observed in the lumenal space between the peripodial and disc proper cell layers, consistent with previous reports where $\mathrm{HhN}$ has been shown to traverse the lumen [14,22]; interestingly, at least some of the lumenal HhN-GFP was present as punctate structures (data not shown). Quantitative analysis of total HhN-GFP particle distribution in these experiments was not possible since the extracellular accumulation in the shits ${ }^{t 1}$ mutant prevented reliable identification of individual particles. Nonetheless, visual inspection of the HhN-GFP distribution clearly indicates that Shi-dependent endocytosis is

Table 2: ANOVA calculated P-values for significance

\begin{tabular}{lccc}
\hline & Median & $\begin{array}{c}90^{\text {th }} \text { percentile } \\
\text { distance }\end{array}$ & \% within 8 um \\
\hline HhNp vs HhN & $<0.0001$ & $<0.0001$ & $<0.0001$ \\
HhNp vs HhNp(shits $)$ & 0.1887 & 0.0131 & 0.0417 \\
& & & \\
$8 \mathrm{hr}$ vs $24 \mathrm{hr}$ & 0.0009 & 0.0011 & 0.0131 \\
$24 \mathrm{hr}$ vs $72 \mathrm{hr}$ & 0.8641 & 0.1341 & 0.7693 \\
\hline
\end{tabular}

There was no significant interaction between the time factor and the genotype factor. Therefore, the significance of the main effects (time irrespective of genotype or genotype irrespective of time) are reported. P-values less than 0.05 are considered significant.

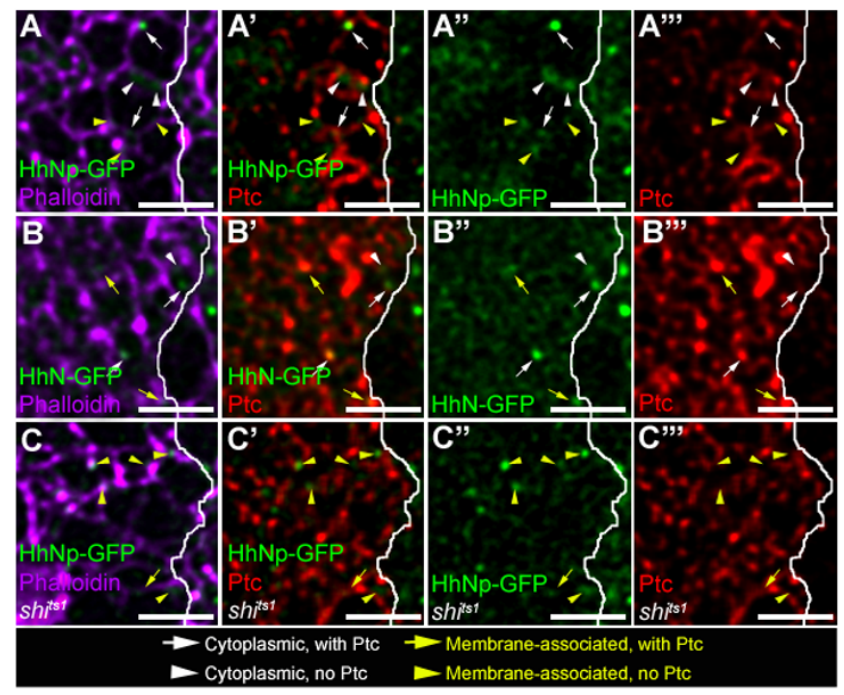

Figure 6

Non-Ptc containing Hh-GFP particles require cholesterol but not endocytosis. (A-C) Ptc co-localization with HhNp-GFP (A), HhN-GFP (B), and HhNp-GFP in the shitsI background (C) after expression induced for $8 \mathrm{hr}$. (A-C) HhGFP (green) labeled with Phalloidin (purple). ( $\left.A^{\prime}-C^{\prime}\right)$ Hh-GFP (green) labeled with Ptc (red). (A"-C") Hh-GFP only. ( $A^{\prime \prime \prime-}$ $\mathrm{C}^{\prime \prime \prime)}$ Ptc only. 4 classes of HhNp-GFP particles are seen: nonPhalloidin associated (cytoplasmic) with Ptc (white arrow), non-Phalloidin associated (cytoplasmic) without Ptc (white arrowhead), Phalloidin (membrane) associated with Ptc (yellow arrow), Phalloidin (membrane) associated without Ptc (yellow arrowhead). Most HhNp-GFP particles are membrane-associated and do not contain Ptc, but cytoplasmic particles have a relatively even distribution with and without Ptc. More HhN-GFP also localizes with Phalloidin, and almost all cytoplasmic HhN-GFP particles contain Ptc. HhNp-GFP particles in shits I mutant background are Phalloidin-associated and many do not contain Ptc. The A/P boundary is marked by a solid white line. Scale bar: $5 \mu \mathrm{m}$. 
not essential for movement of HhN-GFP across the anterior compartment and that much higher levels of apical $\mathrm{HhN}$-GFP accumulate in the absence of Shi function (Figure $4 \mathrm{G}-\mathrm{H}$ ). These results suggest that most HhN-GFP is apically secreted and then degraded via Shi-dependent endocytosis. However, the presence of some basolateral HhN-GFP in these experiments indicates that not all $\mathrm{HhN}$-GFP is apically secreted.

\section{Ptc-negative punctate structures require cholesterol and endocytosis}

In all of the experiments using inducible Hh-GFP, punctate staining patterns were observed. These particles were classified into four groups using Phalloidin, which labels cortical actin near the cell surface, and an antibody specific for the Hh receptor Ptc (Figure 6, Additional Files 6 and 7). Approximately 60 percent of induced HhNp-GFP or HhN-GFP particles in the anterior compartment of wild type discs were at or near the cell surface and classified as "surface-associated" (Table 3); a substantial fraction of these particles were associated with Ptc. Within the 40 percent of HhNp-GFP that was cytoplasmic, there was an even distribution of HhNp-GFP cytoplasmic vesicles with and without Ptc (Table 4). In contrast, nearly all cytoplasmic HhN-GFP was associated with Ptc, suggesting that the cholesterol modification could mediate Ptc-independent endocytosis (Table 4). However, we cannot exclude the possibility that the Hh vesicles without Ptc could arise from Hh dissociating from Ptc after internalization.

In shits1 animals, very few cytoplasmic HhNp-GFP particles could be detected ( 6 percent of total, Table 3 ) and none of them appeared to co-localize with Ptc (Table 4). This result confirms that inhibition of Shi blocks most endocytosis of Hh, including all Ptc-dependent endocytosis. The number of HhNp-GFP that does not co-localize with Ptc is also reduced. We cannot definitively conclude whether the remaining Ptc-negative Hh-GFP particles classified as cytoplasmic are due to incomplete cell surface labeling with Phallodin or represent cytoplasmic particles formed by a Ptc- and Shi-independent mechanism.

Since inhibition of Shi blocks most or all cytoplasmic particles (Table 3), but leads to greater movement through the anterior compartment (Table 1), our results indicate that transcytosis does not play a major role in spreading HhNp-GFP. In addition, we have observed a potential Ptcindependent mechanism for HhNp-GFP uptake that is dependent on both the cholesterol modification and endocytosis.

\section{Discussion \\ Hh-GFP distribution and gradient formation}

We have generated inducible and functional GFP-tagged versions of full length and $\mathrm{N}$-terminal $\mathrm{Hh}$, which allows us to study newly synthesized Hh movement and distribution in live samples, as well as fixed tissues. Our initial analysis of HhNp-GFP localization in living tissue demonstrated similar localization to endogenous $\mathrm{HhNp}$, in particles that were mostly endosomes in the anterior. Upon close examination of HhNp-GFP and HhN-GFP distribution, both were found in punctate structures that localized more apically in the anterior compartment, although basolateral structures were also observed. We have also seen that the Hh gradient appears to require a minimum of 24 hours to fully form. We approximate the minimum HhNp rate of movement to be at least $1 \mu \mathrm{m} /$ hour $\left(8 \mu \mathrm{m}\right.$ distance of the $90^{\text {th }}$ percentile over the first 8 hours) and the $90^{\text {th }}$ percentile distance at $12 \mu \mathrm{m}$, at the furthest time point. This rate of Hh distribution is a minimum calculated rate, and is slower than the reported rate of Decapentaplegic (Dpp) gradient formation of 6-8 hours [44] and the speed of Activin diffusion of $300 \mu \mathrm{m}$ in a few hours [45]. However, more time points are needed to determine the exact rate of Hh gradient formation for comparison to diffusion or transcytosis rates.

\section{HhNp gradient formation through planar diffusion}

Previous studies used a block in endocytosis to try to separate the mechanisms of diffusion, which should not require endocytosis, and transcytosis, which should require endocytosis. We expressed wild type Shi in the posterior cells of $s h i^{t s} 1$ mutant discs and studied diffusion versus transcytosis of a pulse of newly produced Hh. This enabled a simultaneous block of endocytosis in the target cells and production of $\mathrm{Hh}$ to examine $\mathrm{Hh}$ movement. Unexpectedly, we observed HhNp-GFP particles even though we had blocked endocytosis. Upon closer examination, almost all of these particles were associated with the cell surface and not cytoplasmic, indicating these were

Table 3: Hh-GFP co-localization with Ptc in wing discs

\begin{tabular}{|c|c|c|c|c|c|c|}
\hline & \multicolumn{3}{|c|}{ Cytoplasmic } & \multicolumn{3}{|c|}{ Surface-associated } \\
\hline & \%Total & \%Ptc & $\%$ no Ptc & \%Total & $\%$ Ptc & \%no Ptc \\
\hline $\mathrm{HhNp-GFP}$ & $40 \pm 7$ & $22 \pm 7$ & $18 \pm 2$ & $60 \pm 8$ & $16 \pm 6$ & $44 \pm 7$ \\
\hline $\mathrm{HhN}$-GFP & $40 \pm 12$ & $37 \pm 11$ & $3 \pm 2$ & $60 \pm 12$ & $22 \pm 8$ & $38 \pm 11$ \\
\hline $\mathrm{HhNp-GFP(shitsI)}$ & $6 \pm 2$ & 0 & $6 \pm 2$ & $94 \pm 2$ & $27 \pm 1$ & $67 \pm 1$ \\
\hline
\end{tabular}

3 samples were counted for each genotype. Phalloidin co-localization was counted as surface-associated. 
Table 4: Non-membrane-associated Hh-GFP co-localization with Ptc in wing discs

\begin{tabular}{lcc}
\hline & $\begin{array}{c}\text { \% Co-localized with } \\
\text { Ptc }\end{array}$ & $\begin{array}{c}\text { \% } \\
\text { Not co-localized with } \\
\text { Ptc }\end{array}$ \\
\hline HhNp-GFP & $55 \pm 7$ & $45 \pm 7$ \\
HhN-GFP & $92 \pm 3$ & $8 \pm 3$ \\
HhNp-GFP(shis 1$)$ & 0 & 100 \\
\hline
\end{tabular}

3 discs were counted for each genotype

not intracellular transcytotic vesicles. This suggests that cycles of vesicular endocytosis and exocytosis are unlikely to contribute to movement of HhNp. The absence of HhNp-GFP particles in the lumen when endocytosis was blocked indicates that wild-type Hh has restricted planar movement, as previously demonstrated [14]. Statistical analysis indicated that the median values were not significantly different whereas the further distance of the $90^{\text {th }}$ percentile and the lower percentage of vesicles within 8 $\mu \mathrm{m}$ was statistically significant (Table 2 ). This indicates that while endocytosis is required for properly shaping the gradient, particularly near the A/P boundary, it is not required for $\mathrm{HhNp}$ to move into the anterior compartment. This also suggests that the differences in HhNp-GFP distribution in wild type versus shits 1 mutant background are not as dramatic as HhNp-GFP versus HhN-GFP. However, small differences can still have a significant effect on signaling, particularly when local concentrations of $\mathrm{HhNp}$ are important in cell fate determination $[28,34]$. These observations support the model of HhNp-GFP distribution via planar diffusion.

\section{Cholesterol is required for the steep HhNp gradient}

We observed that HhN-GFP was able to travel three times faster than HhNp-GFP ( $27 \mu \mathrm{m}$ versus $8 \mu \mathrm{m}$ distance of the $90^{\text {th }}$ percentile over the first 8 hours) and to target more distant cells in the anterior compartment ( $31 \mu \mathrm{m}$ distance of the $90^{\text {th }}$ percentile at the furthest time point), consistent with earlier observations that $\mathrm{HhN}$ had a longer range than HhNp $[14,19,20]$. HhNp-GFP has a steep gradient with a sharp decline and the cholesterol is required for forming this steep gradient, as demonstrated by the higher percentage of HhNp-GFP particles within the first $8 \mu \mathrm{m}$ from the expressing cells than HhN-GFP. HhNp activates higher levels of the short range target genes en and ptc $[14,20]$, and this suggests that the purpose of the cholesterol modification is to restrict $\mathrm{HhNp}$ closer to the expressing cells, resulting in a precise region of short range target gene activation.

HhN-GFP without the cholesterol was also able to travel into the anterior compartment in the absence of endocytosis. When endocytosis is blocked, HhN-GFP was found to accumulate at high levels in the extracellular lumenal space between the disc proper and the peripodial layer.
This observation is similar to a previous report [14], suggesting that HhN-GFP is free to diffuse three-dimensionally, and again evidence that the cholesterol acts to restrict HhNp movement.

Previous studies have observed that $\mathrm{HhN}$ is capable of being secreted from the peripodial cells and signals to the columnar epithelial cells $[14,22]$. However, we believe that peripodial HhN-GFP has a minimal contribution on the distribution profile. Since the posterior compartment of the peripodial membrane overlies the section that the measurement data originated from, we attempted to take the contribution from the peripodial membrane into account by subtracting the $\mathrm{HhN}$ signal from the end of the distribution profile from the rest of the data set. Subtracting this signal did not significantly alter our results and conclusions.

\section{Ptc-negative vesicles}

Further examination of the Hh-GFP particles detected four classes of particles including Ptc-negative cytoplasmic vesicles. Interestingly, we observed that HhNp-GFP has a significantly higher percentage of these Ptc-negative particles than HhN-GFP. Previous studies have also observed intracellular Ptc-negative Hh vesicles and shown that they are still present in cells mutant for ptc [14,34,35,37]. However, there are distinct differences in our observations from previous studies. Previous results showed that most of HhNp co-localizes with Ptc internally $[14,34]$ while a high proportion of $\mathrm{HhN}$ does not co-localize with Ptc [14]. Since these studies were done at the Hh gradient steady state, the Ptc receptor could have been saturated by this point, resulting in higher levels of HhNp and Ptc colocalization [14,34]. Additionally, clones looking at $\mathrm{HhN}$ and Ptc co-localization appear to be outside of the high Ptc-expressing stripe [14] and since there is less Ptc there, one might conclude that there is less Ptc co-localization.

The Ptc-negative vesicles could represent HhNp-GFP that has somehow dissociated from Ptc after internalization, possibly as a part of a recycling mechanism. C. elegans Hhrelated peptides are sorted to multivesicular bodies (MVBs), then recycled back to the apical surfaces for secretion [46]. However, a recent study reported that Hh does not go through the Rab11-mediated recycling pathway [22]. Another possibility is the presence of another receptor besides Ptc, such as the low density lipoprotein receptor, Megalin, previously demonstrated to interact with vertebrate Shh [47]. The absence of these vesicles when endocytosis is blocked indicates that they are not essential for transport although further studies of these Ptc-negative vesicles are required to elucidate the nature of these vesicles. 


\section{Conclusion}

Previous publications have reported that cholesterol modification of Hh is important for its distribution by analyzing target gene expression. However, these studies showed discrepancies in the range of non-cholesterol modified $\mathrm{HhN}$ and in the apicobasal localization of the different forms of Hh leading to different models of Hh distribution. We have developed a system to induce a pulse of newly synthesized Hh that can be used to further characterize formation of the Hh gradient. Inducible expression, where a pulse of newly synthesized protein is generated, would enable observations of movement during gradient formation instead of at the gradient steady-state, and at protein concentrations closer to endogenous levels. Since contradictory observations exist about the mechanisms regulating morphogen distribution and gradient formation not only for Hh but also for Dpp and Wingless (Wg), clarification could come from using an inducible system together with quantitative measurements.

We have observed that $\mathrm{HhN}$ can be detected at a longer range than modified $\mathrm{HhNp}$. We have quantitatively demonstrated that newly synthesized HhNp-GFP distribution requires cholesterol and can occur without endocytosis in agreement with published results. Additionally, HhNpGFP is detected in intracellular vesicles that do not colocalize with Ptc but we conclude that they are not essential for Hh distribution since they are not observed in endocytosis defective cells. Furthermore, in our inducible system, the modified and unmodified forms of Hh localize at both apical and basolateral regions suggesting there may not be a preferential region for movement. Our data support a model where the cholesterol modification of $\mathrm{Hh}$ is required to restrict its planar diffusion, thereby forming a steep gradient.

\section{Methods}

\section{Drosophila stocks and genetic experiments}

The following mutants and transgenes have been previously described: $h h^{G S 1}$, an amorphic allele also known as $h h^{11}$ [48]; shits $[49]$, UAS-shi+ also known as UAS-dynamin [44], tubulin-Gal80ts ${ }^{2}$ [43], UAS-dsRed [50], UAS-GFPdally-like [28], UAS-ptc-YFP [51], Hh-Gal4 [40], Ptc-Gal4 [52], and 71B-Gal4 [38].

UAS- $h$ hF-GFP and UAS- $h h N-G F P$ transgenic flies were generated. These fusion proteins are similar to those previously described [34,37]. To construct UAS-hhF-GFP, GFP was inserted in frame into full length $h$ h between amino acids $254(\mathrm{H})$ and $255(\mathrm{~V})$. The PCR primers used are as follows: GAGTCGCGGCCGCATCATGGATA and ATGGATCCGTGGGAACTGATCGACGAATC for the first half of full length $h h(h h 1)$; ACGGATCCATGGTGSGCAAGGGCGAG and ACGAATTCCTTGGTACAGCTCGTCATGCC for GFP; AGGAATTCGTGCACGGCTGCTTCAC and TGGGTACCCAGGATTCCATCATCAAT for the second half of full length $h h(h h 2)$. PCR fragments were generated using $h$ h cDNA and eGFP-N1 (Clontech) plasmids as templates, and cloned into pBluescript (Stratagene) in the following restriction enzymes sites (underlined in PCR primer sequence): NotI/BamHI for $h h 1$, BamHI/EcoRI for GFP, and EcoRI/KpnI for hh2. The full hhF-GFP (hh1-GFPhh2) sequence was then cloned into pUASp2 [53] using the NotI/KpnI restriction enzyme sites. To construct UAS$H h N-G F P$ that lacks the cholesterol modification, $h h$ was truncated at amino acid 257 (G) and GFP was cloned in frame immediately behind truncated $h h$. The PCR primers used were: GAGGTACCGAGAAACAGCAAACAACGAGTCTTAG and ATGGATCCAAGCCGTGGGAACT for $h h N$. The HhN PCR fragment was cloned into pUASp that already contained GFP using KpnI/BamHI restriction enzyme sites (underlined in PCR primer sequence). All PCR fragments were sequenced (Macrogen). Each construct was co-injected with the delta 2-3 transposase helper plasmid into $\mathrm{w}^{1118}$ embryos to generate transgenic lines.

For rescue and localization experiments, the following larval genotypes were used:

\section{En-Gal4; UAS-hhF-GFP $h h^{\mathrm{GS} 1}$}

UAS-dsRed/+; UAS-hhF-GFP/Hh-Gal4

UAS-hhF-GFP/71B-Gal4

UAS- $h h F / 71 B-G a l 4$

UAS-CD8-GFP/+; 71B-Gal4/+

UAS-myrpalm-CFP; UAS-hhF-GFP/Hh-Gal4

UAS-ptc-YFP/Ptc-Gal4

Ptc-Gal4/+; UAS-GFP-Dlp

For experiments analyzing Hh temporal distribution, the following larval genotypes were generated:

Hsflp UAS-dsRed/+; UAS-hhF-GFP $h h^{G S 1} / H h-G a l 4$ tubGal80ts $^{2}$

Hsflp UAS-dsRed/+; UAS-hhN-GFP $h h^{G S 1} / H h-G a l 4$ tubGal80ts $^{2}$

To examine Hh distribution in discs with an endocytosisdefect in the anterior compartment, a shi mutant allele was used. Shi is the Drosophila homologue of mammalian GTPase Dynamin and the shits 1 mutant allele is a temperature sensitive allele with the permissive temperature at $18^{\circ} \mathrm{C}$ and the restrictive temperature at $32^{\circ} \mathrm{C}$. These larvae have a shi mutant background at the restrictive temperature which coincides with the expression of wild-type Shi under the Gal80-Gal4 system to rescue the mutant pheno- 
type in the posterior compartment. For these experiments, the following larval genotypes were generated:

shits ${ }^{1}$ FRT19A; UAS-shi+/Hsflp UAS-dsRed; UAS-hhF-GFP hhGS1/Hh-Gal4 tub-Gal80ts ${ }^{2}$

$s h i^{t s 1}$ FRT19A: UAS-shi+/Hsflp UAS-dsRed; UAS- $h h N-G F P$ $h h^{G S 1} /$ Hh-Gal4 tub-Gal80ts ${ }^{2}$

Induction of Hh-GFP expression using Gal80ts:

Larvae were raised at $18^{\circ} \mathrm{C}$. Third instar larvae (day 10-15 at the Gal 80 permissive temperature $18^{\circ} \mathrm{C}$ ) were reared at $32^{\circ} \mathrm{C}$ (the Gal80 restrictive temperature) for 8,24 , or 72 hours. Larvae were dissected at room temperature, and fixed immediately before immunostaining (total time between removal from permissive temperature to fixation was 5-10 minutes).

\section{Imaginal Disc Preparation and Immunostaining}

Larvae were removed from $32^{\circ} \mathrm{C}$ to room temperature, dissected, and fixed immediately (total time of 5-10 minutes). Immunostaining was performed according to Patel [54]. For induction studies, the following modifications were used. Briefly, discs were fixed in $4 \%$ paraformaldehyde in PBS for 20 minutes, washed in PBS, blocked for 30 minutes in PBS with $0.5 \%$ BSA and 5\% normal goat serum (NGS), incubated in primary antibody for 1 hour in PBS with $0.1 \%$ TritonX-100, 0.5\% BSA, and 5\% NGS, washed in PBS for 20 minutes, incubated in secondary antibody diluted in the initial blocking solution for 30 minutes, and washed for 30 minutes. Discs were mounted in $50 \%$ glycerol/PBS. Strips of double-stick tape were added to the slides as spacers to prevent compression of the discs.

Primary antibodies were used at the following concentrations: rat anti-DCAD 1:50 [55]; mouse anti-Ptc 1:50 [56], rabbit anti-dsRed 1:500 (Clontech), rat anti-Ci 1:10 [57], mouse anti-GFP 1:250 (Molecular Probes). Secondary antibodies used were anti-rabbit and anti-rat Alexa 555 1:1000, anti-mouse Alexa 647 1:1000 (Molecular Probes), and anti-rabbit Cy3 1:600 (Jackson Laboratories).

Protocols for membrane labeling, endosome labeling, and extracellular labeling have been described previously $[41,44,58]$. Briefly, to label membranes, discs were mounted in $9 \mu \mathrm{M}$ FM4-64 (Molecular Probes) diluted in $1 \times$ PBS and incubated for 20 minutes at $25^{\circ} \mathrm{C}$ before live imaging. To label endocytic compartments, discs were incubated with $17 \mu \mathrm{M}$ tetramethylrhodamine-dextran (3000 MW, Molecular Probes) diluted in incomplete M3 media for 10 minutes in the dark at $25^{\circ} \mathrm{C}$, washed, and mounted in incomplete M3 media, and incubated for 30 minutes at $25^{\circ} \mathrm{C}$ before live imaging. For extracellular labeling, discs were incubated with anti-GFP (1:250 dilution in incomplete M3 media) for 30 minutes on ice before being washed $5 \times$ with ice cold $1 \times$ PBS and fixed in $4 \%$ paraformaldehyde/PBS for 20 minutes at room temperature. Subsequent processing is the same as stated above for immunostaining. For Alexa 546 and Alexa 647 Phalloidin (Molecular Probes) labeling, Phalloidin was diluted 1:40 in blocking solution and added during secondary antibody incubation step for 20-30 minutes before washes and mounting.

\section{Microscopy, Image Acquisition, and Analysis}

Fluorescence images were collected on a Leica TCSSP2 AOBS confocal microscope, and processed using the Leica Confocal Software 2.5 Build 1347, Adobe Photoshop 7.0, AutoDeBlur \& AutoVisualize X 1.4.1 (MediaCybernetics) and Imaris 5.0.1 (Bitplane).

To count Hh-GFP containing vesicles, $79.35 \mathrm{um}^{2} \mathrm{XY}$ sections were collected using the $63 \times$ objective, in the center of the wing pouch every $0.5 \mu \mathrm{m}$ for the entire depth of the disc (60-100 $\mu \mathrm{m}$ which was approximately $120-200$ sections). All discs were imaged under identical microscope settings for laser power, pinhole, and gain. Quantitative analysis was done in the Imaris software program.

\section{Vesicle identification and distance measurement}

For negative controls (the 0 hour time point), surface intensity thresholds were set just below background levels. This resulted in some background to be incorrectly identified as real signal. After this step, surfaces were sorted according to volume and any surface with a volume of less than $0.03 \mathrm{um}^{3}$ was discarded, leaving only a few background surfaces. This strategy was used to maximize true Hh-GFP signal identification and minimize incorrect identification of background signal.

We applied this strategy to Hh-GFP expressing samples. Surface intensity thresholds were set to just below background, then sorted by volume, and surfaces with volumes more than $0.03 \mu \mathrm{m}^{3}$ were counted and measured.

After vesicle identification, a "Distance Transformation" tool generated a distance map from the UAS-dsRed signal marking the expressing cells. This map was applied to the surfaces to determine the shortest distance of vesicles from the Hh expressing cells. The distance measurements were then imported into Excel and plotted to generate distribution profiles. For each sample, total particle numbers were normalized by dividing the number of particles at each distance by the total number of particles for that sample. The normalized data was then averaged to generate the overall distribution profile. 


\section{Statistical Analysis}

To determine whether the measurement values of median, $90^{\text {th }}$ percentile distance, and percentage at $8 \mu \mathrm{m}$ were significant between genotypes and/or time points, the measurements were subjected to the analysis of variance (ANOVA). The natural log of the medians, natural log of the $90^{\text {th }}$ percentile distances and the raw values for percentage at $8 \mu \mathrm{m}$ were analyzed as these met the normality assumptions of the ANOVAs. Specifically, the Tukey's HSD test was used to determine significance. There was no significant interaction between the time factor and the genotype factor. Therefore, the significance of the main effects (time irrespective of genotype or genotype irrespective of time) are described. P-values less than 0.05 are considered significant.

\section{Quantification of Ptc and Phalloidin co-localization}

Particles were identified in the same way as for distance measurement quantification. After particle identification, each particle was analyzed through the z-stack for colocalization with Ptc and Phalloidin, then sorted into the appropriate category.

\section{Western Blot}

Using the $71 B$-Gal4 drivers to express various transgenes, salivary glands were dissected from the following larvae: $w^{1118}$ (10 glands), UAS-CD8:GFP (5 glands), UAS- $h h N$ GFP $h h^{G S 1}$ (10 glands), UAS- $h h F-G F P ~ h h^{G S 1}$ (10 glands), and UAS- $h h F$ (15 glands) and put on ice. Salivary glands were put in $40 \mu \mathrm{L}$ of sample buffer and broken up with a Dounce Homogenizer. The lysate was spun down and the supernatant was collected and loaded on a $10 \%$ polyacrylamide gel for SDS-PAGE. The blot was first labeled for presence of GFP, then stripped and re-probed for a tubulin loading control. Antibodies used were rabbit anti-GFP 1:1500 (Molecular Probes), mouse anti-tubulin 1:3000 (Oncogene), and anti-rabbit and anti-mouse HRP 1:20000 (Jackson Laboratories).

\section{Wing Preparations}

Wings were collected from adult flies expressing various transgenes under the control of the 71B-Gal4 driver. Whole flies were put in isopropanol, wings were pulled off fly bodies and mounted in 50\% Canada Balsam/isopropanol.

\section{Authors' contributions}

VFS generated the constructs, carried out the genetics, immunofluorescence and localization studies, quantitative analysis, and drafted the manuscript. KAJ performed the Western blot analysis and generated constructs. MB participated in experimental design, coordination, and writing of the manuscript. IT conceived the study, participated in its design, coordination, and writing of the manuscript.

\section{Additional material}

\section{Additional File 1}

Extracellular Hh localizes apically in particles and basolaterally in particles and along the membrane. (A-B) Ptc-YFP (green, A) and GFP-Dlp (green, B), and extracellular labeling (red). As controls for the extracellular labeling protocol with the anti-GFP antibody, Ptc-YFP was used as a negative control since YFP is attached to the cytoplasmic region of Ptc and GFP-Dlp was used as a positive control since GFP is attached to the extracellular region of Dlp. (C-D) HhNp-GFP (green), extracellular labeling with anti-GFP (red), and DCAD to mark the apical region (blue- $C, C^{\prime \prime}$, $D, D^{\prime \prime}$; purple-C'", $\left.D^{\prime \prime \prime}\right)$. Two separate examples of extracellular HhNpGFP, extracellular HhNp-GFP is detected in the anterior apically in particles (arrows in C and D), and basolaterally both in particles (arrowheads in $C$ and D) and with a membrane association (bracket in $C$ and D).

Click here for file

[http://www.biomedcentral.com/content/supplementary/1471213X-7-43-S1.tiff]

\section{Additional File 2}

Hh gradient forms by $24 \mathrm{hr}$ of induction. (A-D) Induced expression of $H h N p-G F P(A-B)$ and HhN-GFP (C-D) in wild-type background. (A-D) $25 \mu \mathrm{m}$ projections; $\left(A^{\prime}-D^{\prime}\right) 20 \mu \mathrm{m} Z$-section projections. 24 and $72 \mathrm{hr}$ distribution of HhNp-GFP appears similar, also seen for HhN-GFP. Scale bar: $5 \mu \mathrm{m}$

Click here for file

[http://www.biomedcentral.com/content/supplementary/1471213X-7-43-S2.tiff]

\section{Additional File 3}

Individual histograms of raw data with median, $90^{\text {th }}$ percentile distance and $\%$ within $8 \mu \mathrm{m}$ values. (A) HhNp-GFP at $8 \mathrm{hr}$ time point: $n=5$. (B) HhNp-GFP at $24 \mathrm{hr}$ time point: $n=16$. (C) HhNp-GFP at $72 \mathrm{hr}$ time point: $n=5$. (D) HhN-GFP at $8 \mathrm{hr}$ time point: $n=4$. (E) HhN-GFP at $24 \mathrm{hr}$ time point: $n=7$. (F) HhN-GFP at $72 \mathrm{hr}$ time point: $n=6$. (G) HhNp-GFP in shits1 mutant background at $8 \mathrm{hr}$ time point: $n=4$. (H) HhNp-GFP in shits 1 mutant background at $24 \mathrm{hr}$ time point: $n=7$. Click here for file

[http://www.biomedcentral.com/content/supplementary/1471213X-7-43-S3.tiff]

\section{Additional File 4}

Full distribution profiles of Hh-GFP. (A-C) Mean of normalized HhNpGFP (green) versus $H h N$-GFP (red) distribution profiles in a wild-type background at 8 (A), 24 (B), and $72 \mathrm{hr}(\mathrm{C})$ time points. All samples were normalized to generate percentages of particles at the distances. Normalized data was then averaged to generate distribution profiles. More HhNpGFP is found closer to the A/P boundary ( 0 on the $x$-axis) than HhN-GFP at $8 \mathrm{hr}(A), 24 \mathrm{hr}(B)$, and $72 \mathrm{hr}(C)$ time points. (D-E) Mean of normalized HhNp-GFP distribution profiles in wild-type background (green) versus shits1 mutant background (blue). More HhNp-GFP is also found closer to the $\mathrm{A} / \mathrm{P}$ boundary ( 0 on the $x$-axis) in the wild-type background than in the shits1 mutant background at 8 (D) and $24 \mathrm{hr}(E)$. The same $H h N p-G F P$ distribution profiles in the wild-type background from $A$ and $B$ are used for $D$ and $E$, respectively.

Click here for file

[http://www.biomedcentral.com/content/supplementary/1471213X-7-43-S4.tiff] 


\section{Additional File 5}

Constitutively expressed HhNp-GFP accumulates at basal membranes after blocking endocytosis. (A-D) HhNp-GFP (green) localization prior to (A) and after an $8 \mathrm{hr}(B-D)$ endocytosis block in the shits1 mutant background with Phalloidin (red) as a cell surface marker; $3 \mu \mathrm{m}$ Z-section projections. HhNp-GFP does not normally accumulate at cell surfaces in the anterior compartment ( $\mathrm{A} / \mathrm{P}$ boundary is marked by a solid white line). At the $\mathrm{shi}^{\mathrm{t} \mathrm{1}}$ permissive temperature, HhNp-GFP accumulates primarily at the basal cell surfaces in the anterior to varying degrees (B-high, C-intermediate, D-low). Scale bar: $5 \mu \mathrm{m}$

Click here for file

[http://www.biomedcentral.com/content/supplementary/1471213X-7-43-S5.tiff]

\section{Additional File 6}

Quantification scheme of Hh-GFP membrane localization and co-localization with Ptc. (A) Hh-GFP surfaces were generated to identify particles based on the same criteria used in particle distance measurements. Each particle was individually located for particle classification (white arrow connected to box). (B-D) Classification of particles. After particle identification, Hh-GFP particles (green) were located in $X Y, X Z$, and $Y Z$ views (B). Co-localization was determined with Phalloidin (purple, C) and Ptc (red, D) in these views through the $z$-stack (white arrows identify the same particle in $X Z$ and $Y Z$ views that was originally identified in the $X Y$ view). Scale bar: $5 \mu \mathrm{m}$

Click here for file

[http://www.biomedcentral.com/content/supplementary/1471-

213X-7-43-S6.tiff]

\section{Additional File 7}

Non-Ptc containing Hh-GFP particles require cholesterol but not endocytosis. (A-C) Z-section of Ptc co-localization with HhNp-GFP (A), HhNGFP (B), and HhNp-GFP in the shits1 background (C) after expression induced for $8 \mathrm{hr}$. (A-C) Hh-GFP (green) labeled with Phalloidin (purple). ( $\left.A^{\prime}-C^{\prime}\right) H h$-GFP (green) labeled with Ptc (red). ( $\left.A^{\prime \prime}-C^{\prime \prime}\right)$ Hh-GFP only. (A'"'-C'") Ptc only. 4 classes of HhNp-GFP particles are seen: nonPhalloidin associated (cytoplasmic) with Ptc (white arrow), non-Phalloidin associated (cytoplasmic) without Ptc (white arrowhead), Phalloidin (membrane) associated with Ptc (yellow arrow), Phalloidin (membrane) associated without Ptc (yellow arrowhead). Most HhNp-GFP particles are Phalloidin-associated and do not contain Ptc, but cytoplasmic particles have a relatively even distribution with and without Ptc. More HhN-GFP also localizes with Phalloidin, and almost all of the cytoplasmic HhN-GFP particles contain Ptc. HhNp-GFP particles in shi ${ }^{\text {ts } 1}$ mutant background are Phalloidin-associated and many do not contain Ptc. The A/P boundary is marked by a solid white line. Scale bar: $5 \mu \mathrm{m}$

Click here for file

[http://www.biomedcentral.com/content/supplementary/1471213X-7-43-S7.tiff]

\section{Acknowledgements}

We thank Pernille Rorth for the pUASp2 vector; Marcos Gonzalez-Gaitan for the UAS-dynamin fly stock; Phil Beachy for the UAS-hhF stock; Matt Scott for the UAS-ptc-YFP fly stock; Xinhua Lin for the UAS-GFP-dlp fly stock; Isabel Guerrero for the anti-Ptc antibody; Bob Holmgren for the anti-Ci antibody, Eicke Latz for technical advice on the confocal microscope, Stephen Baker for assistance with statistical analysis. We thank the UMass fly community for helpful discussions. We also thank Tony lp, Adri Thomas, and Sander van den Heuvel for comments on the manuscript. Work in the laboratory of MB was supported by a Research Scholar Grant from the American Cancer Society and a New Scholar in Aging Award from the Ellison Medical Foundation. Work in the laboratory of IT was supported by grants from the National Institutes of Health (ROI GM66220), American Heart Association (Award\# 0230083), and the Concern Foundation.

\section{References}

I. Ingham PW, McMahon AP: Hedgehog signaling in animal development: paradigms and principles. Genes Dev 200I, I5(23):3059-3087.

2. Heemskerk J, DiNardo S: Drosophila hedgehog acts as a morphogen in cellular patterning. Cell 1994, 76(3):449-460.

3. Strigini M, Cohen SM: A Hedgehog activity gradient contributes to AP axial patterning of the Drosophila wing. Development 1997, 1 24(22):4697-4705.

4. Hooper JE, Scott MP: Communicating with Hedgehogs. Nat Rev Mol Cell Biol 2005, 6(4):306-3I7.

5. Tabata T, Takei Y: Morphogens, their identification and regulation. Development 2004, I3 I(4):703-7|2.

6. Lee J], Ekker SC, von Kessler DP, Porter JA, Sun BI, Beachy PA: Autoproteolysis in hedgehog protein biogenesis. Science 1994, 266(5 I90): 1528-1537.

7. Porter JA, Ekker SC, Park WJ, von Kessler DP, Young KE, Chen $\mathrm{CH}$, Ma Y, Woods AS, Cotter RJ, Koonin EV, Beachy PA: Hedgehog patterning activity: role of a lipophilic modification mediated by the carboxy-terminal autoprocessing domain. Cell 1996, 86(I):2I-34.

8. Porter JA, Young KE, Beachy PA: Cholesterol modification of hedgehog signaling proteins in animal development. Science 1996, 274(5285):255-259.

9. Amanai K, Jiang J: Distinct roles of Central missing and Dispatched in sending the Hedgehog signal. Development 200I, I 28(24):5I | 9-5। 27.

10. Chamoun Z, Mann RK, Nellen D, von Kessler DP, Bellotto M, Beachy PA, Basler K: Skinny hedgehog, an acyltransferase required for palmitoylation and activity of the hedgehog signal. Science 200I, 293(5537):2080-2084.

II. Lee JD, Treisman JE: Sightless has homology to transmembrane acyltransferases and is required to generate active Hedgehog protein. Curr Biol 200 I, I I (14): I I 47-I I 52.

12. Micchelli CA, The I, Selva E, Mogila V, Perrimon N: Rasp, a putative transmembrane acyltransferase, is required for Hedgehog signaling. Development 2002, I 29(4):843-85I.

13. Pepinsky RB, Zeng C, Wen D, Rayhorn P, Baker DP, Williams KP, Bixler SA, Ambrose CM, Garber EA, Miatkowski K, Taylor FR, Wang EA, Galdes A: Identification of a palmitic acid-modified form of human Sonic hedgehog. J Biol Chem 1998, 273(22): I4037-I 4045.

14. Callejo A, Torroja C, Quijada L, Guerrero I: Hedgehog lipid modifications are required for Hedgehog stabilization in the extracellular matrix. Development 2006, I33(3):47|-483.

15. Chen MH, Li YJ, Kawakami T, Xu SM, Chuang PT: Palmitoylation is required for the production of a soluble multimeric Hedgehog protein complex and long-range signaling in vertebrates. Genes Dev 2004, I 8(6):64I-659.

16. Gallet A, Rodriguez R, Ruel L, Therond PP: Cholesterol modification of hedgehog is required for trafficking and movement, revealing an asymmetric cellular response to hedgehog. Dev Cell 2003, 4(2): $191-204$.

17. Feng J, White B, Tyurina OV, Guner B, Larson T, Lee HY, Karlstrom RO, Kohtz JD: Synergistic and antagonistic roles of the Sonic hedgehog $\mathbf{N}$ - and C-terminal lipids. Development 2004, I3 I(17):4357-4370.

18. Zeng X, Goetz JA, Suber LM, Scott WJ Jr., Schreiner CM, Robbins DJ: A freely diffusible form of Sonic hedgehog mediates longrange signalling. Nature 200I, 4 I I (6838):716-720.

19. Burke R, Nellen D, Bellotto M, Hafen E, Senti KA, Dickson BJ, Basler $K$ : Dispatched, a novel sterol-sensing domain protein dedicated to the release of cholesterol-modified hedgehog from signaling cells. Cell 1999, 99(7):803-8I5.

20. Dawber RJ, Hebbes S, Herpers B, Docquier F, van den Heuvel M: Differential range and activity of various forms of the Hedgehog protein. BMC Dev Biol 2005, 5:21.

21. Li $Y$, Zhang H, Litingtung $Y$, Chiang C: Cholesterol modification restricts the spread of Shh gradient in the limb bud. Proc Natl Acad Sci U S A 2006, 1 03(17):6548-6553. 
22. Gallet A, Ruel L, Staccini-Lavenant L, Therond PP: Cholesterol modification is necessary for controlled planar long-range activity of Hedgehog in Drosophila epithelia. Development 2006, I33(3):407-4|8.

23. Gofflot F, Hars C, Illien F, Chevy F, Wolf C, Picard JJ, Roux C: Molecular mechanisms underlying limb anomalies associated with cholesterol deficiency during gestation: implications of Hedgehog signaling. Hum Mol Genet 2003, I 2(I 0): I I87-I I 98.

24. Lewis PM, Dunn MP, McMahon JA, Logan M, Martin JF, St-Jacques B, McMahon AP: Cholesterol modification of sonic hedgehog is required for long-range signaling activity and effective modulation of signaling by Ptcl. Cell 200I, I 05(5):599-6I2.

25. Bellaiche $Y$, The I, Perrimon N: Tout-velu is a Drosophila homologue of the putative tumour suppressor EXT-I and is needed for Hh diffusion. Nature $1998,394(6688): 85-88$

26. Bornemann DJ, Duncan JE, Staatz W, Selleck S, Warrior R: Abrogation of heparan sulfate synthesis in Drosophila disrupts the Wingless, Hedgehog and Decapentaplegic signaling pathways. Development 2004, I 3 I(9): 1927-1938.

27. Desbordes SC, Sanson B: The glypican Dally-like is required for Hedgehog signalling in the embryonic epidermis of Drosophila. Development 2003, I 30(25):6245-6255

28. Han C, Belenkaya TY, Wang B, Lin X: Drosophila glypicans control the cell-to-cell movement of Hedgehog by a dynaminindependent process. Development 2004, I 3 I(3):60 I-6I I.

29. Lum L, Yao S, Mozer B, Rovescalli A, Von Kessler D, Nirenberg M Beachy PA: Identification of Hedgehog pathway components by RNAi in Drosophila cultured cells. Science 2003 299(56 I 5):2039-2045.

30. Takei Y, Ozawa Y, Sato M, Watanabe A, Tabata T: Three Drosophila EXT genes shape morphogen gradients through synthesis of heparan sulfate proteoglycans. Development 2004, I 3 | ( I):73-82.

31. The I, Bellaiche $\mathrm{Y}$, Perrimon N: Hedgehog movement is regulated through tout velu-dependent synthesis of a heparan sulfate proteoglycan. Mol Cell I999, 4(4):633-639.

32. Bumcrot DA, Takada R, McMahon AP: Proteolytic processing yields two secreted forms of sonic hedgehog. Mol Cell Biol I995, I 5(4):2294-2303.

33. Chen $Y$, Struhl G: Dual roles for patched in sequestering and transducing Hedgehog. Cell 1996, 87(3):553-563.

34. Torroja C, Gorfinkiel N, Guerrero I: Patched controls the Hedgehog gradient by endocytosis in a dynamin-dependent manner, but this internalization does not play a major role in signal transduction. Development 2004, I 3 I ( I 0):2395-2408.

35. Gallet A, Therond PP: Temporal modulation of the Hedgehog morphogen gradient by a patched-dependent targeting to lysosomal compartment. Dev Biol 2005, 277(I):5I-62.

36. Wendler F, Franch-Marro X, Vincent JP: How does cholesterol affect the way Hedgehog works? Development 2006, | 33( I 6):3055-306 |.

37. Gorfinkiel N, Sierra J, Callejo A, Ibanez C, Guerrero I: The Drosophila ortholog of the human Wnt inhibitor factor shifted controls the diffusion of lipid-modified hedgehog. Dev Cell 2005, 8(2):24I-253.

38. Brand $\mathrm{AH}$, Perrimon $\mathrm{N}$ : Targeted gene expression as a means of altering cell fates and generating dominant phenotypes. Development 1993, I I 8(2):40 I-4I5.

39. Ingham PW: Transducing Hedgehog: the story so far. Embo 1998, I 7( I 3):3505-35II.

40. Tanimoto $H$, Itoh $S$, ten Dijke $P$, Tabata $T$ : Hedgehog creates a gradient of DPP activity in Drosophila wing imaginal discs. Mol Cell 2000, 5(I):59-7I.

41. Strigini M, Cohen SM: Wingless gradient formation in the Drosophila wing. Curr Biol 2000, I 0(6):293-300.

42. McClure KD, Schubiger G: Developmental analysis and squamous morphogenesis of the peripodial epithelium in Drosophila imaginal discs. Development 2005, I32(22):5033-5042.

43. McGuire SE, Le PT, Osborn AJ, Matsumoto K, Davis RL: Spatiotem poral rescue of memory dysfunction in Drosophila. Science 2003, 302(565I): I 765-I768.

44. Entchev EV, Schwabedissen A, Gonzalez-Gaitan M: Gradient for mation of the TGF-beta homolog Dpp. Cell 2000 | 03(6):98|-99|
45. Gurdon JB, Harger P, Mitchell A, Lemaire P: Activin signalling and response to a morphogen gradient. Nature 1994 37 I (6497):487-492.

46. Liegeois S, Benedetto A, Garnier JM, Schwab Y, Labouesse M: The V0-ATPase mediates apical secretion of exosomes containing Hedgehog-related proteins in Caenorhabditis elegans. Cell Biol 2006, I 73(6):949-96I.

47. McCarthy RA, Barth JL, Chintalapudi MR, Knaak C, Argraves WS: Megalin functions as an endocytic sonic hedgehog receptor. J Biol Chem 2002, 277(28):25660-25667.

48. Mohler J: Requirements for hedgehog, a segmental polarity gene, in patterning larval and adult cuticle of Drosophila. Genetics 1988, I 20(4): 106I-1072.

49. Grigliatti TA, Hall L, Rosenbluth R, Suzuki DT: Temperature-sensitive mutations in Drosophila melanogaster. XIV. A selection of immobile adults. Mol Gen Genet 1973, I20(2): I07-I | 4.

50. Kasuya J, Iverson L: personal communication to FlyBase. 2000

5I. Zhu AJ, Zheng L, Suyama K, Scott MP: Altered localization of Drosophila Smoothened protein activates Hedgehog signal transduction. Genes Dev 2003, I 7(I 0): | 240- | 252.

52. Speicher SA, Thomas U, Hinz U, Knust E: The Serrate locus of Drosophila and its role in morphogenesis of the wing imaginal discs: control of cell proliferation. Development 1994 I 20(3):535-544

53. Rorth P: Gal4 in the Drosophila female germline. Mech Dev |998, 78(I-2): ||3-||8.

54. Patel NH: Imaging neuronal subsets and other cell types in whole-mount Drosophila embryos and larvae using antibody probes. Methods Cell Biol 1994, 44:445-487.

55. Oda H, Uemura T, Harada Y, Iwai Y, Takeichi M: A Drosophila homolog of cadherin associated with armadillo and essential for embryonic cell-cell adhesion. Dev Biol I994, I 65(2):7 I6-726.

56. Capdevila J, Pariente F, Sampedro J, Alonso JL, Guerrero I: Subcellular localization of the segment polarity protein patched suggests an interaction with the wingless reception complex in Drosophila embryos. Development 1994, I 20(4):987-998.

57. Motzny CK, Holmgren R: The Drosophila cubitus interruptus protein and its role in the wingless and hedgehog signal transduction pathways. Mech Dev |995, 52(I): | 37-I50.

58. Greco V, Hannus M, Eaton S: Argosomes: a potential vehicle for the spread of morphogens through epithelia. Cell 200I, I 06(5):633-645.

Publish with Biomed Central and every scientist can read your work free of charge

"BioMed Central will be the most significant development for disseminating the results of biomedical research in our lifetime. "

Sir Paul Nurse, Cancer Research UK

Your research papers will be:

- available free of charge to the entire biomedical community

- peer reviewed and published immediately upon acceptance

- cited in PubMed and archived on PubMed Centra

- yours - you keep the copyright 